\title{
Prognostic significance of third ventricle blood volume in intracerebral haemorrhage with severe ventricular involvement
}

\author{
Dimitre Staykov, ${ }^{1}$ Bastian Volbers, ${ }^{1}$ Ingrid Wagner, ${ }^{1}$ Hagen B Huttner, ${ }^{1}$ \\ Arnd Doerfler, ${ }^{2}$ Stefan Schwab, ${ }^{1}$ Juergen Bardutzky ${ }^{1,3}$
}

\begin{abstract}
${ }^{1}$ Department of Neurology, University of Erlangen, Erlangen, Germany

${ }^{2}$ Department of Neuroradiology, University of Erlangen, Erlangen, Germany

${ }^{3}$ Department of Neurology, University of Freiburg, Freiburg, Germany
\end{abstract}

\section{Correspondence to} Dr D Staykov, Department of Neurology, University of Erlangen, Schwabachanlage 6 91054 Erlangen, Germany; dimitre.staykov@uk-erlangen.de

Received 7 November 2010 Revised 9 March 2011

Accepted 13 March 2011

Published Online First

21 April 2011

\begin{abstract}
Background and purpose Intraventricular haemorrhage (IVH) is an independent predictor of poor outcome in spontaneous intracerebral haemorrhage (ICH). Larger IVH volume and increasing number of affected ventricles have been associated with worse prognosis, however, little is known about the prognostic value of blood volume in the different parts of the ventricular system. Therefore, the correlation of IVH volume in the third, fourth and lateral ventricles with outcome in patients with $\mathrm{ICH}$ and severe $\mathrm{IVH}$, treated with intraventricular fibrinolysis (IVF), was investigated.

Methods Patients with ICH $<40 \mathrm{ml}$, severe IVH and
\end{abstract} acute hydrocephalus were treated with IVF. The course of IVH volume for each ventricle was measured by CT based volumetry. Outcome at 90 days was assessed by a telephone follow-up survey and correlated with initial IVH volume.

Results 50 patients aged $62.5 \pm 10.3$ years with spontaneous ICH $(12.5 \pm 10.8 \mathrm{ml})$ and severe IVH $(33.5 \pm 25 \mathrm{ml})$ were included. Clearance of the third and fourth ventricle from blood occurred after $3 \pm 1.9$ days. Initial IVH volume in the third ventricle $(3.8 \pm 3.3 \mathrm{ml})$ was predictive for poor outcome (OR 2.6 per $\mathrm{ml}, \mathrm{p}=0.02$ ). Correlation between larger IVH volume in the fourth ventricle and poor outcome showed a trend towards significance $(p=0.07)$. Total IVH volume and lateral ventricle IVH volume were not correlated with outcome. Conclusion Despite rapid clot removal, initial IVH volume in the third ventricle was a strong and independent negative predictor. This is possibly explained by irreversible damage of brainstem structures by the initial mass effect of IVH.

\section{INTRODUCTION}

Numerous studies, including large recent prospective trials, have identified the presence of intraventricular haemorrhage (IVH) as a strong and independent predictor of higher mortality and poor outcome in intracerebral haemorrhage $(\mathrm{ICH}){ }^{1-3}$ Volume of ventricular blood and extension of the haematoma to all ventricles have been shown to be the strongest risk factors for poor prognosis. ${ }^{3} 4$ However, little is known about the prognostic value of blood volume in the different parts of the ventricular system, particularly in the third and fourth ventricles located within the brainstem structures with high eloquence. Very few studies have investigated in detail the role of IVH with third and fourth ventricle involvement as a cause of morbidity. ${ }^{3} 56$ Two retrospective case series of approximately 20 patients each have suggested that dilation of the third $^{5}$ and fourth ${ }^{6}$ ventricles is associated with worse outcome and high mortality in patients with IVH secondary to spontaneous $\mathrm{ICH}$, especially when obstructive hydrocephalus due to casting of the aqueduct is present. ${ }^{7}$ Another study has identified the presence of blood in the fourth ventricle as a negative prognostic predictor. ${ }^{3}$

Although obstruction of CSF outflow from the ventricles and consecutive hydrocephalus may represent important causes of acute morbidity, the pathophysiological mechanisms of IVH are certainly more complex. As animal studies have demonstrated, the mass effect of the ventricular clot on surrounding brain structures may lead to tissue damage, likely by reduction of local cerebral blood flow. ${ }^{89}$ This pathomechanism may be crucial for clinical prognosis in patients with IVH.

Considering the eloquence of the brainstem, we aimed to investigate if the initial volume of ventricular blood affecting the third and fourth ventricles is related to outcome in patients with $\mathrm{ICH}$, severe IVH and hydrocephalus, who were treated with external ventricular drainage (EVD) and intraventricular fibrinolysis (IVF).

\section{PATIENTS AND METHODS}

The present analysis was performed on unpublished data collected within a prospective study investigating a treatment regimen of IVF with $4 \mathrm{mg}$ of recombinant tissue plasminogen activator (rt-PA) applied every $12 \mathrm{~h}$ followed by early lumbar drainage in patients with severe IVH and hydrocephalus, as previously described in detail. ${ }^{10}$ Since the CLEARIVH dose finding trial has reported a single dose of $1 \mathrm{mg}$ rt-PA applied every $8 \mathrm{~h}$ to have the best safety/efficacy ratio, ${ }^{11}$ we adapted the dose used for IVF, while the study protocol remained identical. For the present analysis, a total of 50 patients were included, 18 treated with $1 \mathrm{mg}$ and 32 treated with $4 \mathrm{mg}$ of rt-PA as a single dose. The study was approved by our institutional ethics committee.

\section{Patient selection}

Patients with spontaneous hypertensive ganglionic $\mathrm{ICH}<40 \mathrm{ml}$, severe ventricular involvement and acute obstructive hydrocephalus due to obstruction of the third and fourth ventricles were included. Exclusion criteria were oral anticoagulant therapy, international normalised ratio on admission $>1.4$, coagulopathy, ICH due to trauma, tumours or vascular malformation, infratentorial ICH, hydrocephalus due to mass effect of the parenchymal 
ICH with blockage of the foramen of Monro, enrolment $>48 \mathrm{~h}$ after symptom onset and age $<18$ years.

\section{Study protocol}

ICH and IVH were diagnosed by cranial CT. CT angiography was performed routinely after initial CT to exclude aneurysm or vascular malformation bleeding. Hydrocephalus was defined by measuring the bicaudate index, considered present if it exceeded the 95th percentile for age, ${ }^{12}$ and evaluating the temporal horn diameter. Glasgow Coma Scale (GCS) was scored on admission. All patients received standard medical treatment according to the EUSI guidelines ${ }^{13}$ and the study protocol.

Acute obstructive hydrocephalus was treated with EVD and intraventriuclar fibrinolysis with rt-PA. In the first group, single rt-PA doses of $4 \mathrm{mg}$ were applied every $12 \mathrm{~h}$ through the ventricular catheter until the third and fourth ventricles were cleared of blood on CT or a maximum cumulative dose of $20 \mathrm{mg}$ was reached. In the second group, a single dose of $1 \mathrm{mg}$ rt-PA applied every $8 \mathrm{~h}$ up to a maximum cumulative dose of $12 \mathrm{mg}$ was used. EVD, lumbar drainage and permanent shunt management followed the study protocol, as previously described in detail. ${ }^{10}$

\section{Neuroimaging data}

Routine cranial CT scans were performed on admission, immediately after EVD placement and then daily up to day 4, on day 7 and day 10 after admission. Each CT scan consisted of 10-12 scull base slices and 10-12 cerebrum slices. The volume (ml) of IVH was calculated by manual tracing of the ventricular haematoma using the freehand region of interest tool of the Syngo Viewer software (Siemens, Erlangen, Germany). The software returned the surface of the traced region of interest in $\mathrm{cm}^{2}$. To receive the volume of the ventricular haematoma, planimetric data were added up and then multiplied by slice thickness: $0.48 \mathrm{~cm}$ for scull base slices and $0.72 \mathrm{~cm}$ for cerebrum slices. Volume of ventricular blood was recorded separately for each ventricle. In a small proportion of patients, MRI follow-up imaging was performed.

\section{Outcome assessment}

A telephone follow-up survey was conducted with patients or their closest relatives at 90 days ( \pm 10 days) after the initial event. Outcome was recorded using the modified Rankin Scale score (mRS) ${ }^{14}$ Good outcome was defined as $\mathrm{mRS} 0-3$ and poor outcome as mRS 4-6.

\section{Statistical analysis}

Statistical analyses were performed using the SPSS 16.0 software package (http://www.spss.com/). The Shapiro-Wilk and Kolmogorov-Smirnov tests were used to analyse distribution of the data. Data are represented as mean $\pm \mathrm{SD}$ or median (range). The Spearman correlation coefficient (Spearman's $\rho$ ) was used to analyse correlations between outcome and independent variables. Univariate logistic regression was used to identify variables with a possible influence on functional outcome at 90 days. Outcome was dichotomised into good (mRS 0-3) and poor (4-6) outcome. Variables that were significant or showed a trend $(p<0.1)$ in the univariate logistic regression models were entered into a multivariate logistic regression model to identify independent predictors of poor outcome. A p value $<0.05$ was considered statistically significant.

\section{RESULTS}

Fifty patients aged $62.5 \pm 10.3$ years were included in the study. Clinical and radiological characteristics are shown in table 1.
Table 1 Clinical and radiological characteristics

\begin{tabular}{ll}
\hline Characteristic ( $\mathbf{n}=\mathbf{5 0})$ & Mean \pm SD or $(\mathbf{n}(\%))$ \\
\hline Age (years) & $62.5 \pm 10.3$ \\
GCS & $10(3-14)$ \\
ICH volume (ml) & $12.5 \pm 10.8$ \\
Total IVH volume (ml) & $33.5 \pm 25$ \\
Lateral ventricles IVH volume (ml) & $26.5 \pm 22.4$ \\
Third ventricle IVH volume (ml) & $3.8 \pm 3.3$ \\
Fourth ventricle IVH volume (ml) & $3.2 \pm 2.7$ \\
Systolic BP on admission (mm Hg) & $163 \pm 23$ \\
Blood glucose on admission (mg/dl) & $154 \pm 62$ \\
NIHSS on admission & $26 \pm 10$ \\
ICH localisation & \\
$\quad$ Putamen/globus pallidus (n (\%)) & $30(60)$ \\
Caudate nucleus (n (\%)) & $7(14)$ \\
$\quad$ Thalamus (n (\%)) & $13(26)$ \\
Intraventricular fibrinolysis & \\
Opening of the third and fourth ventricle (days) & $1.5 \pm 0.6$ \\
Clearance of the third and fourth ventricle (days) & $3.0 \pm 1.9$ \\
\hline
\end{tabular}

BP, blood pressure; GCS, Glasgow Coma Scale; ICH, intracerebral haemorrhage; IVH, intraventricular haemorrhage; NIHSS, National Institutes of Health Stroke Scale.

Ninety days after the initial event, $27 / 50$ patients (54\%) had a good outcome, as defined by mRS $0-3,23 / 50$ patients (46\%) had an unfavourable outcome (death or dependency, mRS 4-6) and four patients had died (8\%).

No complications related to EVD insertion were observed. One patient suffered an asymptomatic intravertricular rebleeding during IVF after receiving a total dose of $8 \mathrm{mg}$ rt-PA. Catheter associated infections (pleocytosis and elevated lactate levels without detection of bacteria) occurred in two patients. The infection was treated successfully with systemic antibiotics.

The course of blood clearance for the third, fourth and lateral ventricles is shown in figure 1. Mean haematoma half life was $1.7 \pm 0.4$ days for the third, $1.7 \pm 0.5$ days for the fourth and $2.7 \pm 1.3$ days for the lateral ventricles.

Spearman's correlation coefficient revealed significant correlations between outcome at 90 days and GCS on admission $(\rho=-0.45, p=0.002)$, initial ICH volume $(\rho=0.4, p=0.006)$, third ventricle IVH volume $(\rho=0.33, p=0.03)$ and fourth ventricle IVH volume $(\rho=0.34, p=0.02)$. A strong trend towards significance was observed for National Institutes of Health Stroke

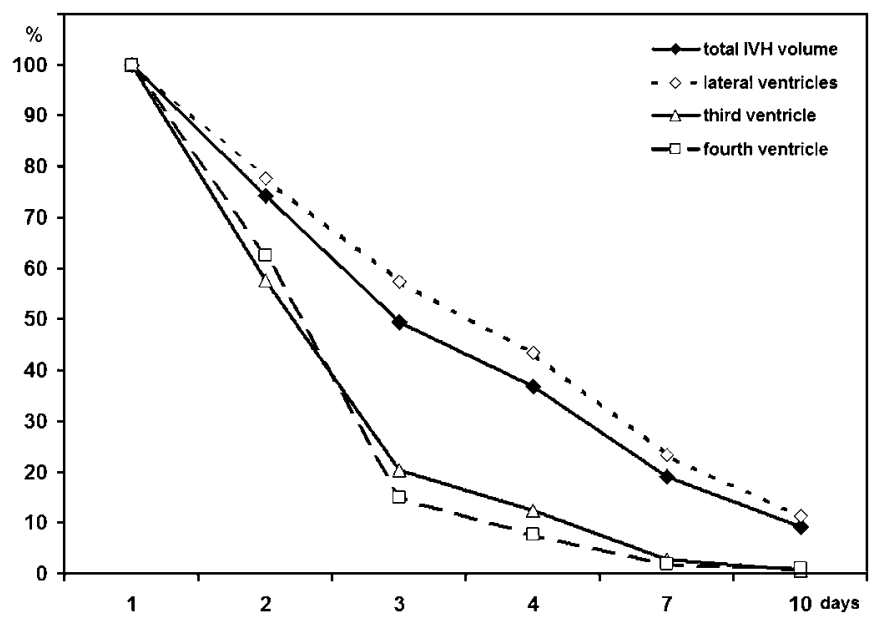

Figure 1 Resolution of intraventricular blood in the course of treatment. Total mean ventricular blood volume and mean blood volume in the lateral, third and fourth ventricles are shown separately as percentage of the corresponding initial volume. IVH, intraventricular haemorrhage. 
Scale (NIHSS) at admission $(\rho=0.25 ; p=0.051)$ and Graeb score $(\rho=0.28 ; p=0.06)$. Outcome at 90 days did not correlate significantly with age, total IVH volume, IVH volume in the lateral ventricles, ICH localisation (putamen/globus pallidus, caudate nucleus, thalamus), clot half-life (for total IVH, or analysed separately), presence of anisocoria on admission, fever or systemic infection, systolic blood pressure or blood glucose on admission. In a partial correlation analysis, IVH volume in the third ventricle was strongly correlated with outcome when GCS and ICH volume were controlled $(r=0.41, p=0.006)$. For the fourth ventricle, the correlation was not significant but there was a trend towards significance $(r=0.28, p=0.07)$.

Univariate logistic regression analyses were performed with dichotomised outcome at day 90 (mRS $0-3$ vs 4-6) for the independent variables systolic blood pressure on admission, blood glucose on admission, presence of fever or systemic infection, NIHSS on admission, GCS, ICH volume, age, IVH volume in the third and fourth ventricles (for each separately), IVH volume in the lateral ventricles and total IVH volume (table 2$)$. The variables showing statistical significance or a trend $(p<0.1)$ (namely, GCS, NIHSS on admission, ICH volume, age, Graeb score and IVH volume in the third and fourth ventricle) were then entered into a multivariate logistic regression model. Only ICH volume, age and IVH volume in the third ventricle showed statistical significance as independent predictors of poor outcome (table 3).

Four patients received MRI follow-up. In those patients, no circumscribed lesions could be described in the periventricular structures surrounding the third and fourth ventricles.

\section{DISCUSSION}

We found that larger initial ventricular blood volume in the third ventricle is a strong and independent predictor of worse outcome in patients with spontaneous ICH and IVH. Furthermore, blood volume in the fourth ventricle showed a trend towards influence on prognosis, while total IVH volume and IVH volume in the lateral ventricles were not associated with outcome. Involvement of the third and fourth ventricles has already been shown to be a negative prognostic predictor in ICH with IVH. ${ }^{3} 56$ However, a volume dependent relationship between initial ventricular haemorrhage in the third ventricle and outcome has not yet been reported.

Table 2 Univariate logistic regression analyses with dichotomised outcome (mRS $0-3$ vs $4-6$ ) as a dependent variable

\begin{tabular}{lll}
\hline & $\mathbf{p}$ Value & ExpB \\
\hline GCS & $\mathbf{0 . 0 4 5}$ & 0.9 \\
ICH volume & $\mathbf{0 . 0 1 5}$ & 1.1 \\
Age & 0.09 & 1.1 \\
Systolic BP on admission & 0.31 & 1.0 \\
Blood glucose on admission & 0.63 & 1.0 \\
Fever/systemic infection & 0.40 & 2.7 \\
NIHSS on admission & 0.053 & 1.1 \\
IVH volume third ventricle & $\mathbf{0 . 0 1 7}$ & 1.5 \\
IVH volume fourth ventricle & $\mathbf{0 . 0 2 5}$ & 1.4 \\
IVH volume lateral ventricles & 0.37 & 1.0 \\
IVH volume (total) & 0.17 & 1.0 \\
Graeb score & 0.052 & 1.5 \\
LD treatment & 0.4 & 2.5 \\
High dose IVF regimen & 0.9 & 0.9 \\
\hline
\end{tabular}

Statistically significant results are highlighted in bold typeface. $\mathrm{BP}$, blood pressure; GCS, Glasgow Coma Scale; ICH, intracerebra haemorrhage; IVF, intraventricular fibrinolysis; IVH, intraventricular haemorrhage; LD, lumbar drainage; mRS, modified Rankin scale; NIHSS, National Institutes of Health Stroke Scale.
Table 3 Multivariate logistic regression analysis

\begin{tabular}{lll}
\hline & $\mathbf{p}$ Value & $\mathbf{O R}(\mathbf{9 5} \% \mathbf{C l})$ \\
\hline GCS & 0.18 & $0.9(0.7$ to 1.08$)$ \\
ICH volume & $\mathbf{0 . 0 1 6}$ & 1.2 (per ml) (1.03 to 1.3) \\
Age & $\mathbf{0 . 0 1 9}$ & $1.2(1.02$ to 1.3$)$ \\
NIHSS on admission & 0.74 & $1.0(0.9$ to 1.08$)$ \\
IVH volume third ventricle & $\mathbf{0 . 0 2}$ & 2.6 (per ml) (1.2 to 5.9) \\
IVH volume fourth ventricle & 0.38 & $0.8(0.5$ to 1.3$)$ \\
Graeb score & 0.80 & $1.1(0.5$ to 2.4$)$ \\
\hline
\end{tabular}

Statistically significant results are highlighted in bold typeface.

GCS, Glasgow Coma Scale; ICH, intracerebral haemorrhage; IVH, intraventricular haemorrhage; NIHSS, National Institutes of Health Stroke Scale.

These findings could possibly be explained by the eloquence of the brainstem structures surrounding the third and fourth ventricles and their functional importance considering, for example, consciousness. The fact that the third and fourth ventricles are anatomically in a key position for the development of acute obstructive hydrocephalus makes the interpretation of such volume effects difficult because even a very small clot in the aqueduct may lead to obstruction with devastating consequences. When such patients are treated with EVD and IVF, clots in the third and fourth ventricles are rapidly dissolved and hydrocephalus, a major factor influencing outcome, ${ }^{7}$ is usually effectively treated. ${ }^{10} 15$ In this setting, pathomechanisms other than obstruction of CSF outflow can be unmasked. As animal studies have shown, those pathomechanisms include the mass effect of the ventricular haematoma with reduction of local cerebral blood flow in the surrounding brainstem tissue. ${ }^{89}$ In the present study, all patients received IVF. 'Opening' of the third and fourth ventricles was achieved after only $1.5 \pm 0.6$ days and total clearance from blood after $3 \pm 1.9$ days, therefore the contact between the blood clot and the brainstem was limited to a relatively short period of time. Despite this, initial IVH volume in the third ventricle still showed the strongest correlation with worse outcome. Therefore, the immediate damage to the brainstem caused by the ventricular clot seems to be considerable and essential for prognosis.

A longer persistence of the ventricular clot-for example, in patients not treated with IVF-may lead to an even stronger negative effect because of the longer exposure of the brainstem to reduced cerebral blood flow and also to the influence of blood and its breakdown products, which have been shown to cause inflammatory damage to ependymal and subependymal brainstem tissue in experimental studies. ${ }^{16} 17$

Interestingly, correlations with outcome were not found when looking at IVH volume in the lateral ventricles although clearance of blood there occurred markedly later in the course of IVF compared with the third and fourth ventricles. This finding may be explained in part by the larger volume of the lateral ventricles with a larger 'buffering capacity' before distension with relevant reduction of local cerebral blood flow. On the other hand, subependymal tissue layers surrounding the lateral ventricles, exposed to blood mediated damage, may be of less functional eloquence in terms of later recovery and influence on outcome.

Despite the relatively high total IVH volume of $33.5 \pm 25 \mathrm{ml}$, it was no longer significantly associated with worse outcome in our analysis while other known negative prognostic predictors such as GCS, age and ICH volume were confirmed, or at least showed a trend. This is a notable finding since IVH and the amount of ventricular blood have been consistently identified as strong predictors of poor outcome in patients with $\mathrm{ICH}^{1-3}$ As already shown, ${ }^{10}$ we aimed to treat IVH at an early stage with 
a specific IVH targeted regimen, consisting of IVF and early lumbar drainage. This more aggressive treatment algorithm for IVH in our study may have contributed to attenuation of the negative impact of total ventricular haematoma on outcome although we were not able to demonstrate a correlation between IVF related parameters (clot half-life) and $\mathrm{mRS}$ at 90 days. When looking at the Graeb score instead of total IVH volume, we observed a strong trend for association between larger scores and worse outcome in the univariate regression analysis but this finding could not be confirmed in the multivariate regression model. These results may be explained by the stronger impact of third and fourth ventricle blood amount on Graeb score, accounting for $33 \%$ of the maximum score of 12 points. This proportion is lower when using actual IVH volumes.

Our study had several limitations. Firstly, all patients had ICH with severe IVH and acute hydrocephalus, and were treated with EVD, IVF and early lumbar drainage. This is a highly selective cohort who received a specific combination treatment. However, this fact strengthens our findings because an even stronger association of blood volume in the third ventricle with worse outcome would be expected without specific IVH targeted therapy. On the other hand, the prognostic impact of IVH volume in different compartments of the ventricular system in a setting without IVF remains unclear. Fourth and lateral ventricle blood volumes may have exerted a greater impact on outcome in untreated patients. Furthermore, in the setting of the present study, the influence of IVH volume and IVF on outcome in patients with a smaller IVH who do not suffer obstructive hydrocephalus also remains unclear.

Secondly, we analysed patients who were treated with different dosing regimens for IVF (4 mg every $12 \mathrm{~h}(\mathrm{n}=32)$ and $1 \mathrm{mg}$ every $8 \mathrm{~h}(\mathrm{n}=18))$. The haematoma resolution in the third and fourth ventricles for both groups was, however, essentially identical when analysed separately.

Thirdly, although the association of ICH volume and unfavourable outcome was present in our study, it was not as strong as that found for IVH in the third ventricle. This finding may be partly explained by the relatively low mean $\mathrm{ICH}$ volume of $12.5 \pm 10.8 \mathrm{ml}$. The low ICH volume and the aggressive IVH targeted treatment in the study may have contributed to the overall low 90 day mortality of only $8 \%$ and the fairly good outcome in the treated cohort (54\% of patients were able to walk independently at that time).

Fourthly, neurological status on admission, scored using the NIHSS, was not correlated to outcome in the multivariate regression analysis; however, a large proportion of patients $(58 \%)$ were already sedated and mechanically ventilated on arrival in our emergency department. Therefore, these patients had higher NIHSS scores, not actually representing their initial neurological status, which was not assessed by the emergency physician. Furthermore, systolic blood pressure on admission, glycaemia and fever or systemic infection did not correlate with outcome in our study. Apart from the high proportion of sedated patients on arrival in the emergency department, the use of antihypertensive drugs and the high prevalence of aspiration pneumonia in the study population, this finding may be at least partly explained by the relatively small sample size.

Finally, the pathophysiological mechanism proposed to explain our findings remains hypothetical because follow-up MRI performed in four patients did not show lesions in the periventricular areas surrounding the third and fourth ventricles. Changes in local cerebral blood flow in the periventricular structures and inflammatory processes caused by blood breakdown products may not be so severe as to cause clearly distinguishable structural damage on $1.5 \mathrm{~T}$ MRI, especially considering the small size of the affected structures. This evidence may still be feasible if higher field MRI (3 Tor even $7 \mathrm{~T}$ ) is used. Furthermore, evaluation of subtle changes may only be possible by means of histological examination and none of the deceased patients underwent autopsy. Therefore, further study on this topic is needed. However, the existing experimental evidence of the harmful effects of IVH on surrounding brain structures 891617 supports our hypothesis.

In conclusion, we identified volume of blood in the third ventricle as a strong and independent predictor of poor outcome in patients with ICH, severe ventricular involvement and hydrocephalus, despite treatment with IVF. This result may possibly be explained by the initial mass effect of the ventricular haematoma on surrounding brainstem structures, leading to immediate irreversible damage.

\section{Competing interests None.}

Ethics approval This study was conducted with the approval of the ethics committee of Erlangen, Germany.

Provenance and peer review Not commissioned; externally peer reviewed.

\section{REFERENCES}

1. Bhattathiri PS, Gregson B, Prasad KS, et al. Intraventricular hemorrhage and hydrocephalus after spontaneous intracerebral hemorrhage: results from the STICH trial. Acta Neurochir Supp/ 2006;96:65-8.

2. Steiner T, Diringer MN, Schneider D, et al. Dynamics of intraventricular hemorrhage in patients with spontaneous intracerebral hemorrhage: risk factors, clinical impact, and effect of hemostatic therapy with recombinant activated factor VII. Neurosurgery 2006;59:767-73

3. Tuhrim S, Horowitz DR, Sacher M, et al. Volume of ventricular blood is an important determinant of outcome in supratentorial intracerebral hemorrhage. Crit Care Med 1999;27:617-21.

4. Broderick JP, Brott TG, Duldner JE, et al. Volume of intracerebral hemorrhage. A powerful and easy-to-use predictor of 30-day mortality. Stroke 1993;24:987-93.

5. Ozdemir 0, Calisaneller T, Hasturk A, et al. Prognostic significance of third ventricle dilation in spontaneous intracerebral hemorrhage: a preliminary clinical study. Neurol Res 2008;30:406-10.

6. Shapiro SA, Campbell RL, Scully T. Hemorrhagic dilation of the fourth ventricle: an ominous predictor. J Neurosurg 1994;80:805-9.

7. Diringer MN, Edwards DF, Zazulia AR. Hydrocephalus: a previously unrecognized predictor of poor outcome from supratentorial intracerebral hemorrhage. Stroke 1998;29:1352-7.

8. Wang YC, Lin CW, Shen CC, et al. Tissue plasminogen activator for the treatment of intraventricular hematoma: the dose-effect relationship. J Neurol Sci 2002;202:35-41.

9. Mayfrank L, Kissler J, Raoofi R, et al. Ventricular dilatation in experimental intraventricular hemorrhage in pigs. Characterization of cerebrospinal fluid dynamics and the effects of fibrinolytic treatment. Stroke 1997;28:141-8.

10. Staykov D, Huttner HB, Struffert T, et al. Intraventricular fibrinolysis and lumbar drainage for ventricular hemorrhage. Stroke 2009;40:3275-80.

11. Hanley D. Final Results CLEARIVH Trial: clot lysis, safety and 30 day functional outcomes. European Stroke Conference, Nice, France, May 13-16, 2008.

12. van Gijn J, Hijdra A, Wijdicks EF, et al. Acute hydrocephalus after aneurysmal subarachnoid hemorrhage. J Neurosurg 1985;63:355-62.

13. Steiner $\mathbf{T}$, Kaste $M$, Forsting $M$, et al. Recommendations for the management of intracranial haemorrhage-part I: spontaneous intracerebral haemorrhage. The European Stroke Initiative Writing Committee and the Writing Committee for the EUS Executive Committee. Cerebrovasc Dis 2006;22:294-316.

14. Bonita R, Beaglehole R. Recovery of motor function after stroke. Stroke 1988;19:1497-500.

15. Staykov D, Huttner HB, Lunkenheimer J, et al. Single versus bilateral external ventricular drainage for intraventricular fibrinolysis in severe ventricular haemorrhage. J Neurol Neurosurg Psychiatry 2010;81:105-8.

16. Mayfrank L, Kim Y, Kissler J, et al. Morphological changes following experimental intraventricular haemorrhage and intraventricular fibrinolytic treatment with recombinant tissue plasminogen activator. Acta Neuropathol (Berl) 2000;100:561-7.

17. Pang D, Sclabassi RJ, Horton JA. Lysis of intraventricular blood clot with urokinase in a canine model: Part 3. Effects of intraventricular urokinase on clot lysis and posthemorrhagic hydrocephalus. Neurosurgery 1986;19:553-72. 

blood volume in intracerebral haemorrhage with severe ventricular involvement

Dimitre Staykov, Bastian Volbers, Ingrid Wagner, Hagen B Huttner, Arnd

Doerfler, Stefan Schwab and Juergen Bardutzky

$J$ Neurol Neurosurg Psychiatry 2011 82: 1260-1263 originally published online April 21, 2011

doi: 10.1136/jnnp.2010.234542

Updated information and services can be found at:

http://jnnp.bmj.com/content/82/11/1260

\section{References \\ Email alerting service}

\section{These include:}

This article cites 16 articles, 6 of which you can access for free at: http://jnnp.bmj.com/content/82/11/1260\#BIBL Receive free email alerts when new articles cite this article. Sign up in the box at the top right corner of the online article.

Topic Collections
Articles on similar topics can be found in the following collections

Hydrocephalus (128)

\section{Notes}

To request permissions go to:

http://group.bmj.com/group/rights-licensing/permissions

To order reprints go to:

http://journals.bmj.com/cgi/reprintform

To subscribe to BMJ go to:

http://group.bmj.com/subscribe/ 